\title{
A Semi-dynamic Evolutionary Power Control Game
}

\author{
Majed Haddad ${ }^{1}$, Eitan Altman ${ }^{2}$, Julien Gaillard ${ }^{1,2}$, and Dieter Fiems ${ }^{3}$ \\ 1 CERI/LIA, University of Avignon, Agroparc BP 1228, Avignon, France \\ 2 INRIA Sophia Antipolis, 10 route des Lucioles, 06902 Sophia Antipolis, France \\ ${ }^{3}$ SMACS Research Group, TELIN Department, Ghent University, Belgium
}

\begin{abstract}
In this paper, we study a power control game over a collision channel. Each player has an energy state. When choosing a higher transmission power, the chances of a successful transmission (in the presence of other interference) increases at the cost of a larger decrease in the energy state of the battery. A central feature in these games is that of the limitation on battery life. Natural questions that arise concern the behavior of mobile users in the presence of interference. How should a mobile user behave given his initial energy state? We study this dynamic game when restricting to simple non dynamic strategies that consist on choosing a given power level that is maintained during the lifetime of the battery. We identify a surprising paradox which we call the Hawk and Dove resource abundance paradox.
\end{abstract}

Keywords: Evolutionary game theory, Hawk and Dove game, Power control, Battery life.

\section{Introduction}

Much research has been devoted to the tradeoff between throughput and power consumption. Yet little has been done concerning another central challenge: the limitation on battery life. In this paper we study power control in a way that combines all above-mentioned aspects. We consider a game among a large population of mobile terminals competing for wireless access. Each terminal attempts transmission. At each attempt, it takes a decision on the transmission power based on its initial battery energy state or level. Higher transmission power improves its throughput, but makes the life of the battery shorter. Each player maximizes his total throughput minus the cost of the transmission over the whole lifetime of his battery. We consider a CDMA type cellular system in which all mobiles transmit simultaneously to a common base station. As the competition involves a large population of mobiles, we find it convenient to model the set of mobiles as continuum in which actions of an individual have a negligible impact on the performance of other mobiles. Each mobile has to take into consideration the initial battery state in the decision.

In this paper, we revisit the power control problem. There is a huge literature on optimization models for controlling transmission power of wireless devices 
sharing a common medium. See [1] for one of the first distributed power control algorithms. We can also cite several articles of Mandayam et al. 2] and references therein that treat the problem of power control considering an energy efficiency non-cooperative game. We use the classical framework of evolutionary games, which we extend to a semi-dynamic context (see below). It deals with large populations in which individuals interact with each other through many local interactions, each of which involve two randomly selected individuals. This pairwise interaction paradigm is relevant for situations of sparse mobile networks in which one may neglect the possibility of simultaneous interference of more than two mobiles.

We consider in this paper a semi-dynamic variant of the well known Hawk and Dove game 3. The latter game has been introduced to describe the evolution of aggressive behavior among animals that compete for food. Variants of the Hawk-Dove (HD) game have been used for various applications in networking. The medium access game considers competition over the access to a common channel through the control of the attempt probabilities 44. The power control game studies the choice of transmission power over a collision channel [5, 6]. Finally, in congestion control the HD game can be used to study the choice between versions of TCP (transmission control protocols) to be used over the Internet [7]. In the HD game, there are two types of individuals: aggressive (Hawk, denoted by H) and peaceful (Dove, denoted by D). In the MAC problem, the aggressive behavior corresponds to a high attempt rate. In power control a Hawk coincides with transmission at a high power, and in the congestion control it is the choice of an aggressive version of TCP (e.g. scalable TCP or high-speed TCP). The standard Hawk and Dove game predicts when one type of behavior ( $\mathrm{H}$ or D) would dominate in the long run, and when we may expect the coexistence of aggressive and peaceful individuals. The equilibrium fraction of each type of behavior is obtained by solving a two-player auxiliary matrix game.

Several authors have studied dynamic variants of this game where individuals are characterized by their energy state [5] 9 A biological variant of this game can be found in 10]. Aggressive behavior requires more energy (which is the case in both the MAC problem as well as in the original HD example). Aggressive behavior requires more energy (which is the case in both the MAC problem as well as in the original HD example). The energy reserve of an individual is defined as the individual state. Thus actions of an individual influence not only the immediate fitness but also the future state of the individual. The objective of an individual is to maximize the total expected fitness during its lifetime. In these dynamic versions of the Hawk and Dove game, the individual strategy is no more a single choice between $\mathrm{H}$ and $\mathrm{D}$, but rather a collection of choices that prescribes how an individual should behave at each possible state.

We consider in this paper a semi-dynamic framework which inherits some features from the static framework and some from the dynamic one. As in the dynamic setting, each player has an individual energy state and the player's action determines not only the immediate fitness but also the future state distribution. Yet in contrast to the dynamic versions of the game, we assume that 
an individual makes state independent choices. The individual chooses $i$ (where $i$ is $H$ or $D$ ) and once the choice is made, the same action $i$ is always used by this individual at any state. The individual is either always aggressive or always peaceful. The problem thus resembles the static one in the fact that the individual has to choose only once, between $\mathrm{D}$ and $\mathrm{H}$. We shall use the two central concepts of evolutionary games. The first is the concept of an Evolutionary Stable Strategy (ESS), which is a distribution of (deterministic or mixed) actions such that, if used, the population is immune against penetration of mutations. This notion is stronger than that of Nash equilibrium as ESS is robust against a deviation of a whole fraction of the population where as the Nash equilibrium is defined with respect to possible deviations of a single player.

The structure of the paper is as follows. In the next Section, we present the system model. In Section 3, we describe the evolutionary game and address the properties of the fitness. Section 4 defines the concept of Evolutionary Stable Strategy. In Section 5, we compute the (pure and mixed) equilibria considering the case with and without breakdown. Discussions about the Hawk and Dove resource abundance paradox are provided in Section 6 . We conclude the paper in Section 7.

\section{Model}

Consider a sparse network that consists of a large population of mobile stations (MSs). Apart from mobile stations, there are also many fix receivers: throw boxes or relays or base stations which we refer to as base stations (BSs) in the remainder. We focus on the case where MSs only transmit when they are in the transmission range of a BS. That is, the situation in which mobiles themselves forward packets of other mobiles is explicitly excluded. As multiple MSs may transmit simultaneously to a BS, interference cannot be avoided. However, assuming that the network is sparse, we do not consider interference between multiple mobiles. This property is relevant to the assumption that the mobile network is sparse, so that one may neglect the possibility of being interfered simultaneously by more than one other mobile. This brings us to the pairwise interaction paradigm of evolutionary games. It is assumed that interactions between individuals occur by some random selection process in which pairs of individuals are selected independently. We consider further that with some probability, when a mobile attempts transmission, there is no interference at all.

We consider two types of terminals: one that transmits at a high power and one that transmits at a low power. We refer to these by Hawks (H) and Doves (D), respectively, thereby referring to the HD game. A mobile user (player) decides which terminal to use, and once this choice is made, he sticks to that choice of terminal for some predetermined time $T$. Considering only pairwise interaction, it is assumed that the sequence of types of terminals with which a given terminal interacts constitutes a sequence of i.i.d. random variables. 
We shall consider two distinct models.

- Model 1 (M1): $T$ is some fix large time, for example two years, which is approximately the expected time until one changes his/her cellular phone. We assume that $T$ does not depend on the type of the phone (H or D).

- Model 2 (M2): $T$ is the time until the mobile runs out of battery power. Note that in this case, $T$ is a function of the choice of type of battery. Indeed, as $\mathrm{H}$ consumes more energy than $D$, it will drain faster.

Models 1 and 2 above have interesting mathematical properties that guarantee the existence of an ESS as described at the end of Section 4 and which in turn facilitate its computation.

We assume that a mobile starts at state $N$. When the battery is empty, it is replaced after a time that corresponds to a geometrically distributed number of transmission opportunities.

We consider in this paper state independent policies, i.e., we consider that the transmission power of a mobile is fixed to always transmit either at the high power $(\mathrm{H})$ or at the lower power $(\mathrm{D})$.

Success probability. Consider a packet transmission of a terminal and let $\delta$ denotes the probability that no other terminal interferes with the transmission. If this is not the case, there is interference between two terminals and the probability that the packet is transmitted successfully is determined by the types of the terminals involved. Let $p_{s}(i, j)$ denote the success probability of the first terminal assuming that this terminal plays $i$ while the other plays $j(i, j \in\{H, D\})$. We have,

$$
p_{s}(i, j)=\left\{\begin{array}{l}
0 \text { for }(i, j)=(D, H), \\
p_{1} \text { for }(i, j)=(D, D), \\
1 \text { for }(i, j)=(H, D), \\
p_{2} \text { for }(i, j)=(H, H) .
\end{array}\right.
$$

Transition probabilities. If the energy level of an individual is $n$ and its action is $D$, then the energy level decreases to $n-1$ with probability $q_{1}$ or it remains unchanged with probability $q_{2}$. We shall have $q_{1}+q_{2} \leq 1$. We shall allow $q_{1}+q_{2}<$ 1 , in which case we assume that there is a positive probability of $1-q_{1}-q_{2}$ for a breakdown which does not depend on the energy level. A breakdown is represented as a transition to an energy state zero.

Analogously, if the energy level of an individual is $n$ and its action is $H$, then the energy level decreases to $n-1$ with probability $q_{3}$ or it remains unchanged with probability $q_{4}$. As for $\mathrm{D}$, we again allow that $q_{3}+q_{4}<1$ in which case we shall have a breakdown probability $1-q_{3}-q_{4}$, a breakdown corresponding to a transition to energy level 0 .

Initial energy level. In the remainder, we assume that a mobile starts at energy level $N_{D}$ or $N_{H}$, depending on the type of mobile. The energy level represents the number of transmissions the mobile can do. With the desire of fairness between 
both populations, we assume that Hawk and Doves have the same amount of energy. However, as transmission by a Hawk requires more energy, a Dove will be able to transmit more times with the same battery, i.e., $N_{D}>N_{H}$ and $q_{1}=q_{3}$. Finally, when the battery is empty, it is immediately replaced.

\section{Properties of the Fitness}

Both Hawks and Doves aim to optimize the amount of data that can be send during the lifetime of the battery, hence the fitness is defined as follows

Definition 1. The long term fitness of a mobile is defined as the sum of the expected number of packets sent by that mobile during the lifetime of its battery. We denote by $V(j, i)$ the long term fitness of a mobile, given that it is of type $j$, and that all others are of type $i$, with $i, j \in\{H, D\}$.

Definition 2. Assume that at any time, a fraction $\alpha$ of the mobiles use action $D$, and the rest use $H$. We then denote by $V(j, \alpha)$ the corresponding long term fitness given that the mobile uses $j$. Moreover, let

$$
V(\beta, \alpha)=\beta V(D, \alpha)+(1-\beta) V(H, \alpha)
$$

be the fitness of a terminal that chooses mobile type $D$ (and always uses it) with probability $\beta$, and otherwise chooses type $H$ (with probability $1-\beta$ ).

We shall motivate these definitions at the end of Section 4. We now mention some properties of the fitness.

(i) Throughout, $\mathrm{H}$ and $\mathrm{D}$ stand both for an action (the power level transmitted at a given time by a given mobile) and a type. The type of a mobile can be interpreted as a state independent pure stationary strategy. Here, a pure stationary strategy is a function that maps states (energy states in our case) to actions.

(ii) Note that the transition probabilities of a user do not depend on the actions of the other users it interacts with. Therefore the total time till a battery drains out is only a function of the mobile's type and not of the actions or types of the other mobiles it interacts with.

(iii) For model M1 (see Section 2), $V(\beta, \alpha)$ can be interpreted as the fitness of a player that uses the mixed strategy $\beta$ given that all the rest uses a mixed strategy $\alpha$. A mixed strategy is a random decision of which type of mobile to use $(\mathrm{H}$ or $\mathrm{D})$; Once the (random) decision is made, we assume that the user stays with this terminal during time $T$.

(iv) The interpretation of (iii) is not valid under assumption M2. Indeed, let $T_{i}$ be the time till the battery empties given that it is of type $i$. Assume that all but one mobile use a mixed strategy $\alpha$. Hence each user chooses to use $D$ until $T_{D}$ with probability $\alpha$. Then the fraction of mobiles that use at a given time an action $D$ is given by

$$
g(\alpha)=\frac{\alpha T_{D}}{\alpha T_{D}+(1-\alpha) T_{H}} .
$$


Conversely, if $\alpha$ stands for the fraction of mobiles that use action $D$, then the fraction of mobiles that are of type $D$ (i.e. that use strategy $D$ ) is given by

$$
\frac{\alpha / T_{D}}{\alpha / T_{D}+(1-\alpha) / T_{H}} .
$$

Remark 1. We assume zero-recharging times in the remainder. Nevertheless, for both models M1 and M2 it is possible to include non-zero recharging times. As for model M2, this requires us to account for the fact that only a fraction of the mobiles is active at a time which affects the fraction of mobiles that use a particular strategy at a time. Again, a function $\hat{g}$ can be introduced which relates the fraction $\alpha$ of mobiles that play $\mathrm{D}$ to the fraction of active mobiles $\hat{g}(\alpha)$ that play $\mathrm{D}$.

\section{Evolutionary Stable Strategies}

\subsection{Nash Equilibrium}

As usual, a symmetric strategy $\alpha$ is a Nash equilibrium if no player can do strictly better by a unilateral deviation to some other pure or mixed action $\beta$.

- For $i=H$ or $i=D, i$ is a pure Nash equilibrium if $V(i, i) \geq V(j, i)$ for $j=H, D$.

- Assuming model M1, we have that $\alpha$ is a mixed Nash equilibrium if $V(\alpha, \alpha) \geq$ $V(\beta, \alpha)$ for all $\beta$.

- Assuming model M2, we have that $\alpha$ is a mixed Nash equilibrium if $V(\alpha, g(\alpha)) \geq$ $V(\beta, g(\alpha))$ for all $\beta$, where $g$ is defined in (2).

An equilibrium is said to be strict if any deviation by any player results in a strictly worse fitness to that player.

\subsection{Definition of a Standard Evolutionary Game}

Suppose that the whole population uses a strategy $q$ and that a small fraction $\epsilon$ (called "mutations") adopts another strategy $p$. Evolutionary forces are expected to select against $p$ if

$$
V(q, \epsilon p+(1-\epsilon) q)>V(p, \epsilon p+(1-\epsilon) q)
$$

Definition 3. A strategy $q$ is said to be an Evolutionary Stable Strategy (ESS) if for every $p \neq q$ there exists some $\bar{\epsilon}_{p}>0$ such that (3) holds for all $\epsilon \in\left(0, \bar{\epsilon}_{p}\right)$.

We shall make use of the following characterization of an ESS [12]:

Theorem 1. A strategy $q$ is an Evolutionary Stable Strategy if and only if $\forall p \neq$ $q$ the following conditions holds:

$$
V(q, q) \geq V(p, q),
$$

and if

$$
V(q, q)=V(p, q) \text { then } V(q, p)>V(p, p) \text {. }
$$


The first condition says that the ESS is a Nash equilibrium in the game that describes the interaction between two players. Conversely, if $q$ is a strict Nash equilibrium in that game then it is an ESS in the evolutionary game.

The second condition, referred to as "Maynard Smith's second condition" 13 states that if $q$ is a Nash equilibrium but not a strict Nash equilibrium (i.e. the fitness of a deviation $p$ from $q$ does as good as $q$ when the rest of the population uses $q$ ), then $q$ can still be an ESS if it has an advantage in that it can invade the mutants strategy $p$. In other words, in a population where every one uses $p$, a small deviation to $q$ does strictly better than everyone using $p$.

Let $V(p, q)$ denote the expected fitness (utility) for a player when playing a mixed policy $p$ and when the fraction of the population that plays each pure strategy $i$ is given by $q(i)$. The expected fitness is then linear in both $p$ and $q$ and can be written as $p \mathbf{V} q^{T}$ where $\mathbf{V}$ is the matrix whose $i, j$ th entry equals $V(i, j)$, and where $p$ (resp. $q$ ) is a row vector whose $i$ th entry is $p(i)$ (resp. $q(i))$. Theorem 1 then states that the ESS of an evolutionary game can be characterized by properties of the equilibria of an auxiliary game. In our case this auxiliary game is the matrix game $\mathbf{V}$. Note that not every matrix game has an ESS.

\subsection{ESS in the Semi-dynamic Game}

Consider the following two pure strategies of a player (i) always play D, and (ii) always play $\mathrm{H}$. With some abuse of notation we denote these policies by $\mathrm{D}$ and $H$. When writing the long term fitness of players as a function of the system parameters, we shall see that the fitness is linear in $p$ and $q$ whereby $p$ are now probabilities over the strategies $H$ and $D$ and not over the actions $H$ and $D$. This means that a mixed strategy is obtained by tossing a coin, and according to the outcome, the player always uses $D$ or always uses $H$. Notice that if we choose between action $D$ and $H$ with some probability $q$ at each time instant, then the expected fitness need not be linear in $q$. This bilinear form of semidynamic games allows us to apply directly the standard theory of evolutionary games to the semi-dynamic case.

Recall that, even though we assume that each individual mobile $j$ always plays the same action, the sequence of actions that are played by the mobiles encountered by some tagged mobile are i.i.d.

While working with mixed strategies allows for directly applying much of the framework of standard evolutionary games, these policies do not allow for an evolution, as once we perform the initial randomized selection between $D$ and $H$, we shall always stick to that choice. Hence, to combine both the flexibility that allows for evolution together with the linear properties of the auxiliary game (the matrix game introduced above), we assume that each mobile uses mixed policies for some limited time $T$, after which a new choice is made and so on. Recall that $T$ either corresponds to the lifetime of the battery (M2) or to the lifetime of the device (M1). Two ways of choosing are given in model M1 and M2, respectively (see beginning of Section 2). 
The definition of $V$ (see definition 2) is suitable for mixed strategies over an infinite time as well as for the finite horizon framework M1. Recall however that for M2 it should be replaced by $V(\beta, g(\alpha))$ where $g$ is given in (2).

\section{Computing the Equilibrium}

Let $V_{n}(i, \alpha)$ denotes the expected fitness of a user who plays $i$ and starts at energy level $n, i, j \in\{H, D\}$. In view of this definition we have, $V(D, \alpha)=V_{N_{D}}(D, \alpha)$ and $V(H, \alpha)=V_{N_{H}}(H, \alpha)$. We find the following recursions for $V_{n}(i, \alpha)$,

$$
\begin{aligned}
& V_{n}(D, \alpha)=\left(\delta+(1-\delta) \alpha p_{1}\right)+q_{1} V_{n-1}(D, \alpha)+q_{2} V_{n}(D, \alpha), \\
& V_{n}(H, \alpha)=(\delta+(1-\delta) \alpha)+(1-\alpha)(1-\delta) p_{2}+q_{3} V_{n-1}(H, \alpha)+q_{4} V_{n}(H, \alpha) .
\end{aligned}
$$

The first equation expresses the total expected fitness of a mobile of type D when starting with $n$ units of energy, till its battery empties. Hence, the equation is composed of two expressions:

(i) The expected fitness corresponding to the current transmission: with probability $\delta$ there is no interference at all so the fitness is one unit. With probability $(1-\delta)$ there is an interaction with another mobile. The fitness equals $p_{1}$ when both mobiles use $D$ which occurs with probability $\alpha$. Otherwise, it is zero.

(ii) The expected fitness collected after the transmission: we first note that with probability $q_{1}$, the energy level after the transmission equals $n-1$, so the expected fitness to go is $q_{1} V_{n-1}(D, \alpha)$. With probability $q_{2}$ the energy level is unchanged so the expected fitness collected after the transmission is $q_{2} V_{n}(D, \alpha)$.

The second equation can be explained following similar lines.

\subsection{With Breakdown}

Solving the recursions for $q_{1}+q_{2}<1$ and $q_{3}+q_{4}<1$ yields,

$$
\begin{aligned}
& V_{n}(D, \alpha)=\frac{\delta+\alpha p_{1} \bar{\delta}}{1-q_{1}-q_{2}}\left(1-\left(\frac{q_{1}}{1-q_{2}}\right)^{n}\right) \\
& V_{n}(H, \alpha)=\frac{\delta+\alpha \bar{\delta}+p_{2} \bar{\delta}(1-\alpha)}{1-q_{3}-q_{4}}\left(1-\left(\frac{q_{3}}{1-q_{4}}\right)^{n}\right),
\end{aligned}
$$

with $\bar{\delta}=1-\delta$ and whereby we assumed $V_{0}(D, \alpha)=V_{0}(H, \alpha)=0$. That is, no fitness can be collected if the battery is empty.

Lemma 1. Assume that both Hawks and Doves are subjected to breakdowns $\left(q_{1}+q_{2} \neq 1\right.$ and $\left.q_{3}+q_{4} \neq 1\right)$, we have for $i \in\{D, H\}$,

$$
V(i, \alpha)=\alpha V(i, D)+(1-\alpha) V(i, H),
$$


with,

$$
\begin{array}{ll}
V(H, H)=\frac{\delta+p_{2}(1-\delta)}{1-q_{3}-q_{4}}\left(1-\left(\frac{q_{3}}{1-q_{4}}\right)^{N_{H}}\right), & V(H, D)=\frac{1}{1-q_{3}-q_{4}}\left(1-\left(\frac{q_{3}}{1-q_{4}}\right)^{N_{H}}\right) \\
V(D, H)=\frac{\delta}{1-q_{1}-q_{2}}\left(\frac{q_{1}}{1-q_{2}}\right)^{N_{D}}, & V(D, D)=\frac{\delta+p_{1}(1-\delta)}{1-q_{1}-q_{2}}\left(\frac{q_{1}}{1-q_{2}}\right)^{N_{D}}
\end{array}
$$

This allows us to express the equilibrium as follows.

Corollary 1. Assuming non-zero breakdown probability, the following holds.

(i) $D$ is a pure equilibrium if

$$
\frac{1}{1-q_{3}-q_{4}} \cdot\left(\frac{q_{3}}{1-q_{4}}\right)^{N_{H}}+\frac{\delta+p_{1}(1-\delta)}{1-q_{1}-q_{2}} \cdot\left(\frac{q_{1}}{1-q_{2}}\right)^{N_{D}}>\frac{1}{1-q_{3}-q_{4}}
$$

(ii) $H$ is a pure equilibrium if

$$
\frac{\delta+p_{2}(1-\delta)}{1-q_{3}-q_{4}} \cdot\left(\frac{q_{3}}{1-q_{4}}\right)^{N_{H}}+\frac{\delta}{1-q_{1}-q_{2}} \cdot\left(\frac{q_{1}}{1-q_{2}}\right)^{N_{D}}<\frac{\delta+p_{2}(1-\delta)}{1-q_{3}-q_{4}}
$$

(iii) Let

$$
\alpha^{*}=\frac{\theta \cdot \frac{\delta}{1-q_{1}-q_{2}}-\rho \cdot \frac{\left(\delta+p_{2}(1-\delta)\right)}{1-q_{3}-q_{4}}}{\theta \cdot \frac{\left((1-\delta)\left(1-p_{2}\right)\right)}{1-q_{3}-q_{4}}-\rho \cdot \frac{\left((1-\delta) p_{1}\right)}{1-q_{1}-q_{2}}}
$$

where $\theta=\left(1-\left(\frac{q_{1}}{1-q_{2}}\right)^{N D}\right)$ and $\rho=\left(1-\left(\frac{q_{1}}{1-q_{2}}\right)^{N H}\right)$. If $\alpha^{*}$ is in the interior of the unit interval then it is a mixed ESS.

\subsection{Without Breakdown}

Assume now that Hawk and Dove are no more subject to breakdown. We have then $q_{1}=1-q_{2}$ and $q_{3}=1-q_{4}$, which yields

$$
\begin{aligned}
V_{n}(D, \alpha) & =\frac{\alpha p_{1}(1-\delta)+\delta}{q_{1}} n \\
V_{n}(H, \alpha) & =\frac{(1-\delta)(1-\alpha) p_{2}+\delta+\alpha(1-\delta)}{q_{3}} n
\end{aligned}
$$

We make the observation that the behavior of the system depends on the ratio $\frac{N_{H}}{N_{D}}$ and not on $N_{D}$ and $N_{H}$ themselves. Let $\gamma=\frac{N_{H}}{N_{D}}$. This allows us to express the equilibrium as follows 
Lemma 2. In the absence of breakdowns $\left(q_{1}=1-q_{2}\right.$ and $\left.q_{3}=1-q_{4}\right)$, we have for $i \in\{D, H\}$,

$$
V(i, \alpha)=\alpha V(i, D)+(1-\alpha) V(i, H)
$$

with,

$$
\begin{aligned}
V(H, H) & =\frac{\gamma\left((1-\delta) p_{2}+\delta\right)}{q_{3}}, & V(H, D) & =\frac{\gamma}{q_{3}} \\
V(D, H) & =\frac{\delta}{q_{1}}, & V(D, D) & =\frac{p_{1}(1-\delta)+\delta}{q_{1}}
\end{aligned}
$$

This gives us the following equilibria.

Corollary 2. In the absence of breakdowns, the following holds.

(i) $D$ is a pure equilibrium if $\gamma<\frac{q_{3}\left(p_{1}(1-\delta)+\delta\right)}{q_{1}}$.

(ii) $H$ is a pure equilibrium if $\gamma>\frac{\delta q_{3}}{\left(p_{2}(1-\delta)+\delta\right) q_{1}}$.

(iii) Let

$$
\alpha^{*}=\frac{\delta q_{3}-q_{1} \gamma\left(p_{2}(1-\delta)+\delta\right)}{(1-\delta)\left(q_{1} \gamma\left(1-p_{2}\right)-q_{3} p_{1}\right)}
$$

If $\alpha^{*}$ is in the interior of the unit interval then it is a mixed ESS.

At the equilibrium, Let $\beta=(\beta, 1-\beta)$ and $\underline{\alpha}=(\alpha, 1-\alpha)$ be two row vectors of probability measures over the available actions $D, H$. Let $\mathbf{V}$ be a matrix whose $(i, j)$ th entry is given by $V(i, j)$. In accordance with Definition 2 , denote by $V(\beta, \alpha)$ the expected fitness of a player who always plays strategy $D$ with probability $\beta$ and always $H$ with probability $1-\beta$, while the fraction of individuals in the population that play $D$ is $\alpha$. We then make the following key observation regarding the total expected utility.

Lemma 3. The expected utility for a player that chooses to be $D$ with probability $\beta$ given that the fraction of $D$ in the population is $\alpha$ can be written in a vector form as

$$
V(\beta, \alpha)=\underline{\beta} \mathbf{V} \underline{\alpha}^{T}
$$

It is thus bilinear. It can therefore be interpreted as the expected fitness for a player in an equivalent one shot game (a symmetric static evolutionary game) where the fraction of $D$ in the population is $\alpha$ and where the player chooses $D$ with probability $\beta$. The equilibria given in Lemma 1 and 2 are ESS. 


\section{The Hawk and Dove Resource Abundance Paradox}

Without loss of generality, we consider the case without breakdown (the case of breakdown is similar). Unless otherwise stated, for all numerical applications, we use the following variables: $p_{1}=0.3, p_{2}=0.1, q_{1}=q_{3}=0.6$.

Figure 1 shows the effect of the parameter of being alone $\delta$ on the mixed ESS equilibrium for several values of $\gamma$. We identify a paradox which we call the Hawk and Dove resource abundance paradox. First, we can see that depending on $\delta$, we have two different behaviors of the mixed ESS: (i) the saturated region: the case where $\delta$ is smaller than a certain threshold $\delta^{*} \approx 5 \%$, and (ii) the nonsaturated region: the case where $\delta>\delta^{*}$. In the non-saturated region, as intuition would suggest, the proportion of Doves at equilibrium increases for increasing $\delta$, and this happens faster as $\delta$ decreases. This can be explained by the fact that in the non-saturated region (low interference), it is more interesting for mobiles to transmit at low power (Dove). This is exactly the opposite for the saturated region where the predominant strategy is Hawk as $\delta$ increases. This is paradoxical since with larger $\delta$, mobiles can get more opportunities to transmit packets successfully with a low power. However, one can find an advantage of being aggressive in this region. In fact, in the saturated regime and for a given $\gamma$, mobiles have incentive to be aggressive (Hawk) since resources are made scarce as the system is highly interfered.

Asymptotically, in a highly interfered system (for $\delta=0$ ) the value for which all the population is Dove $(\alpha=1)$ is given by $\gamma^{*}=\frac{q_{3} p_{1}}{q_{1}}\left(\gamma^{*}=0.3\right.$ in Figure 1) . This is paradoxical because in a highly interfered system, mobiles should behave aggressively in order to have opportunity to transmit!

Moreover, a small increase in $\delta$ gives more opportunities for mobiles to transmit (more resources are made available within the system). The proportion of Hawk at the equilibrium increases faster.

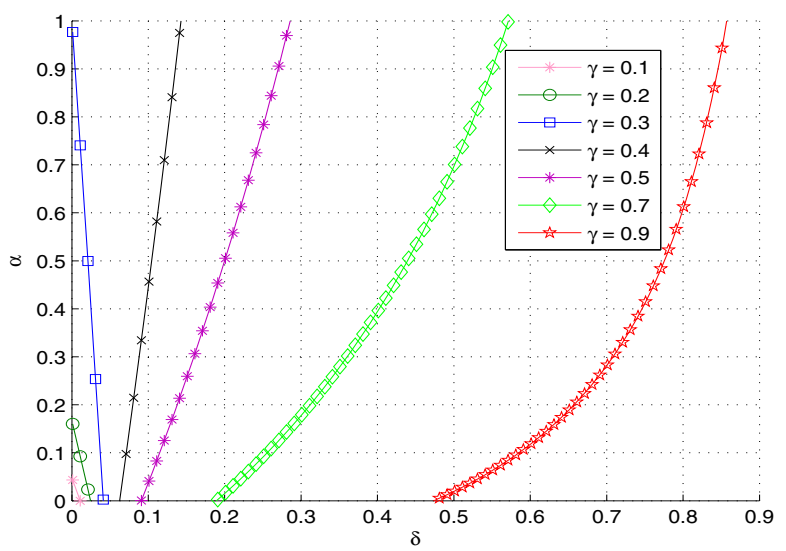

Fig. 1. Variation of the mixed $\operatorname{ESS} \alpha$ for increasing probability of no interference $\delta$ 
Figure 1 also depicts the fact that for low values of $\delta$, the ratio $\gamma$ has more impact on the equilibrium. It means that for a large $\delta$, a change on $\gamma$ will not have a big impact on the equilibrium, whereas with a small $\delta$, a tiny change in $\gamma$ will have enormous consequences on the equilibrium. In particular, one can observe that the more the ratio between initial energy states $\left(N_{H}\right.$ and $\left.N_{D}\right)$ is large, the more the equilibrium is sensitive to a small change in $\delta$.

\section{Conclusion}

We have studied in this paper a semi-dynamic version of the Hawk and Dove game within the framework of evolutionary games. Specifically, we identify a surprising paradox in our Hawk-Dove game namely, the Hawk and Dove resource abundance paradox which offers insights on how mobiles behave in the presence of interference. In this setting, it is shown that at equilibrium, lower probability of no interference has bigger impact on the equilibrium of the power control game.

\section{References}

1. Yates, R.D.: A framework for uplink power control in cellular radio systems. IEEE J. Selected Areas Communications 13(7), 1341-1347 (1995)

2. Goodman, D.J., Mandayam, N.B.: Power control for wireless data. IEEE Personal Communications 7, 48-54 (2000)

3. Maynard Smith, J.: Evolution and the Theory of Games. Cambridge University Press (1982)

4. Tembine, H., Altman, E., El-Azouzi, R.: Delayed Evolutionary Game Dynamics applied to the Medium Access Control. In: Bionetworks 2007, Pisa, Italy (2007)

5. Wiecek, P., Altman, E., Hayel, Y.: Stochastic state dependent population games in wirelesss communications. Transactions on Autoatic Control 56(3), 492-505 (2011)

6. Hayel, Y., Tembine, H., Altman, E., El-Azouzi, R.: A Markov Decision Evolutionary Game for Individual Energy Management. Annals of the International Society of Dynamic Games (2009)

7. Altman, E., El-Azouzi, R., Hayel, Y., Tembine, H.: The evolution of transport protocols: An evolutionary game perspective. Computer Networks 53(10), 17511759 (2009)

8. Altman, E., Hayel, Y.: Markov Decision Evolutionary Games. IEEE Transactions on Automatic Control 55(6) (June 2010)

9. McNamara, J., Merad, S., Collins, E.: The Hawk-Dove Game as an Average-Cost Problem. Avdances in Applied Probability 23(4), 667-682 (1991)

10. Altman, E., Gaillard, J., Haddad, M., Wiecek, P.: Dynamic Hawk and Dove games within flocks of birds. In: Bionetics, New York, England (2011)

11. Cressman, R.: Evolutionary Dynamics and Extensive Form Games. MIT Press (2003)

12. van Veelen, M., Spreij, P.: Evolution in games with a continuous action space. Economic Theory 39(3), 355-376 (2008)

13. Maynard Smith, J.: Game Theory and the Evolution of Fighting. In: On Evolution, pp. 8-28. Edinburgh University Press, Edinburgh (1972) 